\title{
Nonautonomous Bright and Dark Solitons of Bose-Einstein Condensates with Feshbach-Managed Time-Dependent Scattering Length
}

\author{
Qiu-Yan $\mathrm{Li}^{1,2}$, Zai-Dong $\mathrm{Li}^{1,2}, \mathrm{Lu} \mathrm{Li}^{3}$ 因 and Guang-Sheng $\mathrm{Fu}^{2}$ \\ ${ }^{1}$ Department of Applied Physics, Hebei University of Technology, Tianjin 300401, China \\ ${ }^{2}$ School of Information Engineer, Hebei University of Technology, Tianjin, 300401, China and \\ ${ }^{3}$ College of Physics and Electronics Engineering, Shanxi University, Taiyuan, 030006, China
}

\begin{abstract}
We present a family of nonautonomous bright and dark soliton solutions of Bose-Einstein condensates with the time-dependent scattering length in an expulsive parabolic potential. These solutions show that the amplitude, width, and velocity of soliton can be manipulated by adjusting the atomic scattering length via Feshbach resonance. For the cases of both attractive and repulsive interaction, the total particles number is a conservation quantity, but the peak (dip) density can be controlled by the Feshbach resonance parameter. Especially, we investigate the modulation instability process in uniform Bose-Einstein condensates with attractive interaction and nonvanishing background, and clarify that the procedure of pattern formation is in fact the superposition of the perturbed dark and bright solitary wave. At last, we give the analytical expressions of nonautonomous dark oneand two-soliton solutions for repulsive interaction, and investigate their properties analytically.
\end{abstract}

PACS numbers: 03.75.Lm, 05.30.Jp, 67.40.Fd

Keywords: Nonautonomous soliton solution; interaction; Bose-Einstein condensation

\section{INTRODUCTION}

The classical soliton concept was introduced firstly by Zabusky and Kruskal [1] for autonomous nonlinear and dispersive dynamic systems where the time variable has only played the role of an independent variable and has not appeared explicitly in the coefficients of the nonlinear evolution equation. These autonomous solitons do not disperse and completely preserve their localized form and speeds during propagation which has motived a great attention in optical fibers and condensate physics. As a theoretical model, the nonlinear Schrödinger equation has been adopted extensively to govern the dynamics of autonomous bright and dark solitons in optical fibers [2, 3] and Bose-Einstein condensates (BECs) [4].

In BECs bright and dark solitons have been paid more particular interest experimentally and theoretically. The bright soliton [5-11] is expected by the balance between dispersion and attractive mean-field energy. However, collapse of bright soliton [12] may occur owing the attractive interaction of bosons. To avoid this collapse, one should restrict the BECs dynamics into the quasi-onedimensional regime, i.e., the energy of two body interaction is much less than the kinetic energy in the transverse direction. The dark soliton [13-17] can be formed in the case of repulsive interaction of bosons. It denotes the macroscopic excitation characterized by a local density minimum, and a phase gradient of the wave function at the position of the minimum. Comparing with the attractive case, large condensates can be realized for dark soliton owing the repulsive interaction of bosons.

When the physical system is subjected to various external time-dependent forces, the nonautonomous nonlinear

*Electronic address: llz@sxu.edu.cn evolution models typically arise and the term of nonautonomous solitons [18] was introduced firstly. In fact, different aspects of dynamics in nonautonomous models [19 21] and the controllable soliton solutions [18, 2224] in optical fibers have been investigated theoretically. For BECs the nonlinearity resulting from the interatomic interaction is denoted by the effective scattering length which can be tuned experimentally by utilizing the Feshbach resonance 25], even including its sign. A sinusoidal variation of the scattering length has also been used to form patterns such as Faraday waves [26, 27]. The controlling soliton trains [28] was also created starting from periodic waves. Therefore, the BECs solitons forming by magnetically tuning the interatomic interaction near a Feshbach resonance are a typical example of such nonautonomous solitons in external potentials and offer a good opportunity for the nonlinear excitations exploration [29]. The properties of such nonautonomous solitons in BECs are not well explored and it is our purpose in the present paper.

At the mean-field level, the evolution of the macroscopic wave function of BECs can be described by the Gross-Pitaevskii equation [30, 31],

$$
i \hbar \frac{\partial \Psi(\mathbf{r}, t)}{\partial t}=\left[-\frac{\hbar^{2} \nabla^{2}}{2 m}+V_{\text {ext }}(\mathbf{r})+g|\Psi(\mathbf{r}, t)|^{2}\right] \Psi(\mathbf{r}, t),
$$

where $\Psi(\mathbf{r}, t)$ is normalized to the number of condensed atoms, i.e., $N=\int|\Psi|^{2} d^{3} r, m$ is the atomic mass, $V_{\text {ext }}$ is a harmonic trap given by $V_{\text {ext }}(\mathbf{r})=m / 2\left[\omega_{0}^{2} x^{2}+\right.$ $\left.\omega_{\perp}^{2}\left(y^{2}+z^{2}\right)\right]$ with $\omega_{0}$ and $\omega_{\perp}$ being the axial and transverse harmonic oscillator frequencies, and the effective interatomic interaction reads $g=4 \pi \hbar^{2} a_{s} / m$ with $a_{s}$ being the $s$-wave scattering length $\left(a_{s}<0\right.$ for attractive interaction; while $a_{s}>0$ for repulsive interaction). The linear oscillator lengths in the transverse and cigar-axis directions is defined by $l_{\perp}=\left(\hbar / m \omega_{\perp}\right)^{1 / 2}$ and $l_{0}=\left(\hbar / m\left|\omega_{0}\right|\right)^{1 / 2}$, respectively. For a cigar-shaped con- 
densate at a relatively low density, when the energy of two body interactions is much less than the kinetic energy in the transverse direction, i.e. when $\epsilon^{2}=\left(l_{\perp} / \xi\right)^{2} \sim$ $N\left|a_{s}\right| / l_{0} \ll 1$ [31], where $\xi=\left(8 \pi n\left|a_{s}\right|\right)^{-1 / 2}$ is the healing length and $n \propto N /\left(l_{\perp}^{2} l_{0}\right)$ is a mean particle density, then the system becomes effectively quasi-onedimensional regime [5, 32] with the explusive potential

$$
\begin{aligned}
i \frac{\partial}{\partial t} \psi(x, t) & =-\frac{\partial^{2}}{\partial x^{2}} \psi(x, t)-\frac{1}{4} \lambda^{2} x^{2} \psi(x, t) \\
& +2 N \frac{a_{s}}{l_{\perp}}|\psi(x, t)|^{2} \psi(x, t),
\end{aligned}
$$

where $\lambda \equiv 2\left|\omega_{0}\right| / \omega_{\perp} \ll 1$, and the time $t$ and coordinate $x$ has been measured in units $2 / \omega_{\perp}$ and $l_{\perp}$, respectively. In real experiment for BECs [5] the scattering length $a_{s}$ is tuned with a Feshbach resonance from repulsive to attractive. For soliton created with the particle number $N \sim 10^{3}, \omega_{\perp}=2 \pi \times 710 \mathrm{~Hz}, \omega_{0}=2 \pi i \times 20 \mathrm{~Hz}$, the parameter $\lambda=0.056$ is small. With the above conditions, the units $l_{\perp}$ is about $1.44 \mu \mathrm{m}$ and the units time for a BECs trapped with the transversal size order $l_{\perp}$ corresponds to $4.5 \times 10^{-4} \mathrm{~s}$. The lifetime of a BECs is of the order of $1 \mathrm{~s}$, which is about 220 in our dimensionless units. If one chose the scattering length is increased in the form of $a_{s}(t)=a_{0} \exp (\lambda t)$ with the initial scattering length $a_{0}=-0.02 \mathrm{~nm}$. After 40 dimensionless units of time, the value of the atomic scattering length turns to $a_{s}=-0.19$ $\mathrm{nm}$ corresponding to $\epsilon^{2} \sim 0.02$ which provides the safe range parameters.

In terms of the transformation $\psi(x, t)=$ $q(X, T) \exp \left(\lambda t / 2-i \lambda x^{2} / 4\right)$ with $X=x \exp (\lambda t)$ and $T=2 \int_{0}^{t} \exp (2 \lambda \tau) d \tau$, Eq. (2) reduces to the standard form

$$
i \frac{\partial q}{\partial T}+\frac{1}{2} \frac{\partial^{2} q}{\partial X^{2}}-\left(N a_{0} / l_{\perp}\right)|q|^{2} q=0 .
$$

This result show that in BECs the nonautonomous solitons formed by magnetically tuning the interatomic interaction via Feshbach resonance can be obtained from the autonomous solitons. In this paper, we will explore the generalized nonautonomous bright and dark solitons of Eq. (21). As an example, the nonautonomous bright soliton solutions are obtained on the vacuum state background and nonzero background, respectively. With these exact solutions the corresponding dynamic properties are discussed in detail. At last, we investigate the dynamic behavior of the nonautonomous dark soliton solutions.

\section{NONAUTONOMOUS BRIGHT SOLITON SOLUTIONS}

In this section, we consider the case of attractive interaction between atoms, i.e., $a_{0}<0$. In this case, Eq. (3) is a integrable model, and its soliton solutions can be constructed by several technique, such as inverse scattering method 3], Darboux transformation [34 36] and Hirota method [37]. With the expulsive parabolic potential and the Feshbach-managed time-dependent scattering length, we will present the exact nonautonomous bright soliton solutions analytically on the vacuum state background and nonzero background for Eq. (2).

\section{A. Nonautonomous bright soliton solution and} soliton interaction on the vacuum state background

Firstly, we can present the nonautonomous one-soliton solution for Eq. (2) as follows

$$
\psi=A_{s} \operatorname{sech} \theta_{s} \exp \left(i \varphi_{s}+\lambda t / 2\right),
$$

where $\theta_{s}$ and $\varphi_{s}$ are given by

$$
\begin{aligned}
& \theta_{s}=\mu A_{s}\left(x e^{\lambda t}-2 k_{s} \int_{0}^{t} e^{2 \lambda \tau} d \tau\right)-\theta_{0}, \\
& \varphi_{s}=k_{s} x e^{\lambda t}-\frac{\lambda x^{2}}{4}+\left(\mu^{2} A_{s}^{2}-k_{s}^{2}\right) \int_{0}^{t} e^{2 \lambda \tau} d \tau-\varphi_{0},
\end{aligned}
$$

here $\mu=\sqrt{N\left|a_{0}\right| / l_{\perp}}$. The solution $\psi$ in Eq. (44) describes a bright soliton of BECs with time-dependent atomic scattering length in an expulsive parabolic potential, with the initial maximum amplitude $A_{s}$, the initial wave number $k_{s}$, the initial location $\theta_{0} /\left(\mu A_{s}\right)$, and the initial phase $\varphi_{0} / k_{s}$. When $\lambda=0$, Eq. (4) can reduce to the solution for the standard nonlinear Schrödinger equation. From Eq. (5) we see that the soliton in Eq. (41) can undergo compressing effect with the increasing of the scattering length tuned by the Feshbach resonance. This character does not exist for the situation of the uniform nonlinear Schrödinger equation [3]. This nonautonomous soliton has an increase in the peak value, while the particles number is a conservation quantity due to $\int_{-\infty}^{+\infty}\left|\psi_{1-\text { sol }}\right|^{2} d x=2 A_{s} / \mu$. The velocity of soliton is affected by the Feshbach resonance parameter $\lambda$, which reads $V_{s}=-2 k_{s}\left(e^{\lambda t}-\lambda e^{-\lambda t} \int_{0}^{t} e^{2 \lambda \tau} d \tau\right)$. This result show that the size of bright soliton in BECs can be tuned by adjusting the attractive interactions even in the presence of the expulsive potential.

Another interesting problem is to discuss the interaction of two nonautonomous bright solitons. To this purpose we present two-soliton solution of Eq. (2) as follows

$$
\psi=\frac{G_{b}}{F_{b}} \exp (\lambda t / 2)
$$

where

$$
\begin{aligned}
F_{b} & =f_{1} \cosh \left(\theta_{1}+\theta_{2}\right)+f_{2} \cosh \left(\theta_{1}-\theta_{2}\right) \\
& +f_{3} \cos \left(\varphi_{1}-\varphi_{2}\right) \\
G_{b} & =g_{1} \cosh \theta_{1} e^{i \varphi_{2}}+g_{2} \cosh \theta_{2} e^{i \varphi_{1}} \\
& +i g_{3}\left(\sinh \theta_{1} e^{i \varphi_{2}}-\sinh \theta_{2} e^{i \varphi_{1}}\right)
\end{aligned}
$$




$$
\begin{aligned}
& f_{1}=\left(k_{2}-k_{1}\right)^{2}+\mu^{2}\left(A_{2}-A_{1}\right)^{2}, \\
& f_{2}=\left(k_{2}-k_{1}\right)^{2}+\mu^{2}\left(A_{1}+A_{2}\right)^{2}, \\
& f_{3}=-4 \mu^{2} A_{1} A_{2}, \\
& g_{1}=2 A_{2}\left[\left(k_{2}-k_{1}\right)^{2}+\mu^{2}\left(A_{2}^{2}-A_{1}^{2}\right)\right], \\
& g_{2}=2 A_{1}\left[\left(k_{2}-k_{1}\right)^{2}+\mu^{2}\left(A_{1}^{2}-A_{2}^{2}\right)\right], \\
& g_{3}=4 \mu A_{1} A_{2}\left(k_{2}-k_{1}\right),
\end{aligned}
$$

with the parameters

$$
\begin{aligned}
& \theta_{j}=\mu A_{j}\left(x e^{\lambda t}-2 k_{j} \int_{0}^{t} e^{2 \lambda \tau} d \tau\right)-\theta_{0 j}, \\
& \varphi_{j}=k_{j} x e^{\lambda t}-\frac{\lambda x^{2}}{4}+\left(\mu^{2} A_{j}^{2}-k_{j}^{2}\right) \int_{0}^{t} e^{2 \lambda \tau} d \tau-\varphi_{0 j},
\end{aligned}
$$

here $j=1,2, \theta_{0 j}$ and $\varphi_{0 j}$ is an arbitrary real constant, respectively. The solution in Eq. (6) describes a general interaction between two nonautonomous solitons with the different center velocity $V_{s, 1}$ and $V_{s, 2}$, respectively. From Eq. (7) we get the velocity of each soliton

$$
V_{s, j}=-2 k_{s, j}\left(e^{\lambda t}-\lambda e^{-\lambda t} \int_{0}^{t} e^{2 \lambda \tau} d \tau\right), j=1,2 .
$$

In order to understand the nature of two nonautonomous solitons interaction, we analyze the asymptotic behavior of two-soliton solution in Eq. (6). Asymptotically, the solution in Eq. (6) can be written as a combination of two one solutions in Eq. (4). The asymptotic form of two-soliton solution in limits $t \rightarrow-\infty$ and $t \rightarrow \infty$ is similar to that in Eq. (4).

(i) Before collision (limit $t \rightarrow-\infty$ )

(a) Soliton $1\left(\theta_{1} \approx 0, \theta_{2} \rightarrow-\infty\right)$

$$
\psi_{2-\mathrm{sol}} \rightarrow \frac{\gamma_{1} e^{\lambda t / 2}}{2 \sqrt{f_{1} f_{2}}} \frac{e^{i\left(\varphi_{1}+\phi_{1}\right)}}{\cosh \left(\theta_{1}+x_{0}\right)},
$$

(b) Soliton $2\left(\theta_{2} \approx 0, \theta_{1} \rightarrow \infty\right)$

$$
\psi_{2-\mathrm{sol}} \rightarrow \frac{\gamma_{2} e^{\lambda t / 2}}{2 \sqrt{f_{1} f_{2}}} \frac{e^{i\left(\varphi_{2}-\phi_{2}\right)}}{\cosh \left(\theta_{2}-x_{0}\right)} .
$$

(ii) After collision (limit $t \rightarrow \infty$ )

(a) Soliton $1\left(\theta_{1} \approx 0, \theta_{2} \rightarrow \infty\right)$

$$
\psi_{2-\mathrm{sol}} \rightarrow \frac{\gamma_{1} e^{\lambda t / 2}}{2 \sqrt{f_{1} f_{2}}} \frac{e^{i\left(\varphi_{1}-\phi_{1}\right)}}{\cosh \left(\theta_{1}-x_{0}\right)},
$$

(b) Soliton $2\left(\theta_{2} \approx 0, \theta_{1} \rightarrow-\infty\right)$

$$
\psi_{2-\mathrm{sol}} \rightarrow \frac{\gamma_{2} e^{\lambda t / 2}}{2 \sqrt{f_{1} f_{2}}} \frac{e^{i\left(\varphi_{2}+\phi_{2}\right)}}{\cosh \left(\theta_{2}+x_{0}\right)},
$$

where

$$
\begin{aligned}
& \phi_{1}=\arctan \left(g_{3} / g_{2}\right), \phi_{2}=-\arg \left(g_{3} / g_{1}\right), \\
& \gamma_{1}=\sqrt{g_{2}^{2}+g_{3}^{2}}, \gamma_{2}=\sqrt{g_{1}^{2}+g_{3}^{2}}, x_{0}=\frac{1}{2} \ln \left(f_{2} / f_{1}\right) .
\end{aligned}
$$

From the above asymptotic behavior of two-soliton solution, we know that there is no change of the amplitude for each soliton during the process of collision. However, from Eqs. (8) to (11) we find there is a phase exchange $2 \phi_{j}, j=1,2$, and center shift $2 x_{0}$ for soliton 1 and soliton 2 during collision, respectively. These results show that the collision of two nonautonomous solitons is elastic.

\section{B. Nonautonomous bright soliton solution on nonzero background}

It is easy to find two basic solutions of Eq. (2). One is $\psi=0$, which corresponds to the vacuum particle density state. The nonautonomous soliton solutions in Eqs. (4) and (6) are constructed on this zero background to the moment. The other interesting solution of Eq. (2) is a plane wave solution

$$
\psi_{c}=A \exp \left(i \varphi_{c}+\lambda t / 2\right),
$$

with the initial amplitude $A$, wave number $k$, and $\varphi_{c}=$ $-\lambda x^{2} / 4+k x e^{\lambda t}+\left(2 \mu^{2} A^{2}-k^{2}\right) \int_{0}^{t} e^{2 \lambda \tau} d \tau$. This solution can be seen as the background with the temporal variation particle density. It will be very interesting to get the exact soliton solution and its dynamic properties on such background. Employing Darboux transformation [34 [36], we obtain the nonautonomous soliton solution as follows

$$
\psi=\left(A+A_{s} \frac{G_{c}}{\cosh \theta+a \cos \varphi}\right) e^{\frac{1}{2} \lambda t+i \varphi_{c}},
$$

where

$$
\begin{aligned}
G_{c} & =b_{1} \cosh \theta+\cos \varphi+i\left(b_{2} \sinh \theta+c \sin \varphi\right), \\
\theta & =M_{I} x e^{\lambda t}-\left[\mu A_{s} M_{R}+\left(k+k_{s}\right) M_{I}\right] \int_{0}^{t} e^{2 \lambda \tau} d \tau-\theta_{0}, \\
\varphi & =M_{R} x e^{\lambda t}-\left[\left(k+k_{s}\right) M_{R}-\mu A_{s} M_{I}\right] \int_{0}^{t} e^{2 \lambda \tau} d \tau-\varphi_{0},
\end{aligned}
$$

with the parameters $b_{1}=-2 \mu^{2} A A_{s} / D, \quad b_{2}=$ $-2 \mu A M_{R} / D, c=M_{I} /\left(\mu A_{s}\right), D=\mu^{2} A_{s}^{2}+M_{R}^{2}$, and $M_{R}+i M_{I}=\left[\left(k-k_{s}-i \mu A_{s}\right)^{2}+4 \mu^{2} A^{2}\right]^{1 / 2}$, which imply that $M_{I}=0$ as $A_{s}=0$. Here $\theta_{0}, \varphi_{0}, A_{s}, k_{s}, A$, and $k$ is an arbitrary real constant, respectively. From Eq. (13) one can see that, as $A$ vanishes, the solution (13) reduces to one soliton solution in Eq. (44). On the other hand, when the initial amplitude of the soliton $A_{s}$ vanishes, the solution $\psi$ in Eq. (13) reduces to the solution in Eq. (12). Therefore, the exact solution (13) describes generally the dynamics of the nonautonomous bright soliton embedded in the temporal variation particle density background, characterized by the envelop propagation velocity $V_{s c}=\left(\mu A_{s} M_{R} / M_{I}+k+k_{s}\right)\left(e^{t \lambda}-\lambda e^{-t \lambda} \int_{0}^{t} e^{2 \lambda \tau} d \tau\right)-$ $\lambda e^{-t \lambda} \theta_{0} / M_{I}$.

Based on the above exact solution, we analyze in detail the modulation instability process and the formation of 
spatial pattern in BECs. As discussed in Ref. 34, 35], we firstly consider a special case, i.e., $k_{s}=k$. In this situation, there are two representative results:

(i) When $4 A^{2}>A_{s}^{2}$, we have $M_{I}=0$ which implies that the soliton velocity $V_{s c}$ becomes infinite. It is to say that the soliton in Eq. (13) is completely trapped in spatial direction and undergoes the modulation instability process [3]. Indeed, by introducing a small quantity $\epsilon=\exp \left(\theta_{0}\right)$ for $\theta_{0}<0$, and then linearizing with respect to $\epsilon$ we have the approximation for the initial value

$$
\psi(x, 0) \approx\left[\rho+\epsilon \chi \cos \left(\mu M_{1} x-\varphi_{0}\right)\right] e^{-i \lambda x^{2} / 4+i k x},
$$

where $\rho=\left(2 A^{2}-A_{s}^{2}-i A_{s} M_{1}\right) /(2 A)$ with $|\rho|=|A|$, $\chi=A_{s} M_{1}\left(M_{1}-i A_{s}\right) /\left(2 A^{2}\right)$, and $M_{1}=\sqrt{4 A^{2}-A_{s}^{2}}$. The solution of the initial value problem of Eq. (2) can be well described by the solution (13) under the case of $M_{I}=0$. As a result, a small periodic perturbation of the plane wave solution may lead to the onset of instability.

(ii) When $4 A^{2}<A_{s}^{2}$, the soliton (13) becomes

$$
\psi=\left(-A+M_{2} \frac{M_{2} \cos \varphi+i A_{s} \sin \varphi}{A_{s} \cosh \theta-2 A \cos \varphi}\right) e^{\frac{1}{2} \lambda t+i \varphi_{c}},
$$

where $\theta=\mu M_{2}\left(x e^{\lambda t}-2 k \int_{0}^{t} e^{2 \lambda \tau} d \tau\right)-\theta_{0}$ and $\varphi=\mu^{2} A_{s} M_{2} \int_{0}^{t} e^{2 \lambda \tau} d \tau-\varphi_{0}$ with $M_{2}=\sqrt{A_{s}^{2}-4 A^{2}}$. On the nonzero background the nonautonomous bright soliton possesses the same properties as that on zero background. The peak value increases and the soliton width compresses with the increasing value of the scattering length, respectively. The dynamic soliton evolution exhibits the periodic oscillation of the amplitude and breather behavior due to the presence of nonvanishing background [35, 36]. On the other hand, under the effect of the expulsive parabolic potential, the bright soliton can propagate in the longitudinal direction, instead of oscillation in attractive parabolic potential. The atoms of the bright soliton against the background can be obtained, i.e., $\int_{-\infty}^{+\infty}\left(|\psi|^{2}-|\psi( \pm \infty, t)|^{2}\right) d x=A_{s}\left(b_{2}^{2}+c^{2}\right) /\left|M_{I}\right|$ $\int_{-\infty}^{+\infty}\left(A_{s}-2 A \cosh \theta \cos \varphi\right) /\left(\cosh \theta+a_{1} \cos \varphi\right)^{2} d \theta$, where $B=M_{R} / M_{I}, \varphi=B \theta+\Delta$, and $\Delta=\mu A_{s} M_{I}\left(B^{2}+1\right) \int_{0}^{t} e^{2 \lambda \tau} d \tau+B \theta_{0}-\varphi_{0} . \quad$ Вy numerically verifying this integration we find it is a conservation quantity which indicates that during the process of the compression of the bright soliton the number of atoms in the bright soliton keeps invariant. Another interesting problem is how such soliton can be created. From the expression (15), we can see that the initial wave function can be written as the form $\phi=-A e^{i \varphi_{c}} \pm i M_{2} e^{i \varphi_{c}} \operatorname{sech}\left(\mu M_{2} x-\theta_{0}\right)$ as $\varphi_{0}= \pm \pi / 2, \pm 3 \pi / 2, \cdots$. This result shows that the solution (15) can be generated by coherently adding in quadrature a bright soliton to the background.

To better understand the properties of nonautonomous bright soliton on nonvanishing background with the case $k_{s} \neq k$, we can decompose Eq. (13) into the form

$$
\psi=\psi_{d}+\psi_{b}
$$

where

$$
\begin{gathered}
\psi_{d}=\left(A+A_{s} b_{1}+\frac{i A_{s} b_{2} \sinh \theta}{\cosh \theta+a_{1} \cos \varphi}\right) e^{\lambda t / 2+i \varphi_{c}}, \\
\psi_{b}=A_{s} e^{\lambda t / 2+i \varphi_{c}} \frac{\left(1-b_{1}^{2}\right) \cos \varphi+i c \sin \varphi}{\cosh \theta+b_{1} \cos \varphi} .
\end{gathered}
$$

As $A=0$ we have $\psi_{d}=0$ and $\psi_{b}=A_{s} \exp (-i \varphi) \operatorname{sech} \theta_{s}$, which shows that in Eq. (16) the former is zero solution, and the latter gives rise to one soliton solution for Eq. (2). With the increasing of $|A|$, a dip starts to occur for $\psi_{d}$ describing a perturbed grey solitary wave under the effect of nonzero background. At the same time $\psi_{b}$ exhibits the periodic oscillation of the amplitude in propagation which can be considered as a perturbed bright solitary wave. Therefore, the solution (13) describes the superposition of the perturbed dark and bright solitary waves expressing the procedure of the pattern formation.

\section{NONAUTONOMOUS DARK SOLITON SOLUTIONS}

In this section, we consider the case of repulsive interaction between atoms, i.e., $a_{0}>0$. Using the results in Ref. [37, 38], we obtain the nonautonomous dark soliton solution of Eq. (2) in the form

$$
\psi=\frac{\sqrt{\kappa}}{\mu} q(x, t) \exp \left(i \varphi_{d}+\lambda t / 2\right),
$$

where $\varphi_{d}=-\frac{1}{4} \lambda x^{2}+\xi_{0} x e^{\lambda t}-\left(\xi_{0}^{2}+2 \kappa\right) \int_{0}^{t} e^{2 \lambda \tau} d \tau+\zeta_{0}$ and $q(x, t)$ is to be determined. For nonautonomous onesoliton solution, $q(x, t)$ is given by

$$
q(x, t)=\frac{1}{2}\left[\left(1+Z_{1}\right)-\left(1-Z_{1}\right) \tanh \frac{\eta_{1}}{2}\right],
$$

where

$$
\begin{aligned}
\eta_{1} & =P_{1} x e^{\lambda t}+\left(\sqrt{4 \kappa-P_{1}^{2}}-2 \xi_{0}\right) P_{1} \int_{0}^{t} e^{2 \lambda \tau} d \tau+\zeta_{1} \\
Z_{1} & =\frac{\sqrt{4 \kappa-P_{1}^{2}}+i P_{1}}{\sqrt{4 \kappa-P_{1}^{2}}-i P_{1}}
\end{aligned}
$$

From Eq. (19) we see that the existence of nonautonomous dark soliton implies the condition $\kappa \geq P_{1}^{2} / 4$. From the solution (17) with Eq. (18) we clear two special cases, i.e., $Z_{1}= \pm 1$. When $Z_{1}=1$, the nonautonomous dark solitonin Eqs. (17) reduces to plane wave solution $\psi=\sqrt{\kappa} / \mu e^{i \varphi_{d}+\lambda t / 2}$, which corresponds to the variation distribution density of bosons due to the existence of the Feshbach resonance parameter $\lambda$. 
When $\lambda=0$, this case corresponds to the uniform distribution density of bosons. On the other hand, when $Z_{1}=-1$ the solution in Eqs. (17) and (18) becomes $\psi_{1}=-\sqrt{\kappa} / \mu e^{i \varphi_{d}+\lambda t / 2} \tanh \left(\eta_{1} / 2\right)$, where $\eta_{1}=$ $2 \sqrt{\kappa} x e^{\lambda t}-4 \sqrt{\kappa} \xi_{0} \int_{0}^{t} e^{2 \lambda \tau} d \tau+\zeta_{1}$. This solution represents black soliton solution in BECs with variational amplitude and the soliton velocity $V_{d}=2 \xi_{0}\left(e^{\lambda t}-\lambda e^{-\lambda t} \int_{0}^{t} e^{2(\lambda \tau)} d \tau\right)$.

The result in Eqs. (17) and (18) shows that this nonautonomous dark soliton undergo compressing effect with the increasing of the scattering length, while the peak value has an increase. From Eqs. (17) and (18) we have $|q|_{\min }=\sqrt{\kappa-P_{1}^{2} / 4} / \mu \exp (\lambda t / 2)$ which is inverse proportion to the initial soliton width $1 / P_{1}$. The dark soliton possesses the accelerated motion with the absolute increasing scattering length tuned by the Feshbach resonance instead of oscillation in attractive parabolic potential and the velocity reads $V_{d}=\left(2 \xi_{0}-\right.$ $\left.\sqrt{4 \kappa-P_{1}^{2}}\right)\left(e^{\lambda t}-\lambda e^{-\lambda t} \int_{0}^{t} e^{2(\lambda \tau)} d \tau\right)$ obtained from Eq. (19). From Eq. (17) we obtain the particles number in the form $\int_{-\infty}^{+\infty}\left(|\psi|^{2}-|\psi( \pm \infty, t)|^{2}\right) d x=-P_{1} / \mu^{2}$, which is a conservation quantity. The above analysis implies that we can control the dip matter density of dark soliton by adjusting appropriately the Feshbach resonance.

For nonautonomous dark two-soliton solution, the expression of $q(x, t)$ is given by

$$
q_{2}(x, t)=\frac{1+Z_{1} e^{\eta_{1}}+Z_{2} e^{\eta_{2}}+A_{12} Z_{1} Z_{2} e^{\eta_{1}+\eta_{2}}}{1+e^{\eta_{1}}+e^{\eta_{2}}+A_{12} e^{\eta_{1}+\eta_{2}}},
$$

where

$$
\begin{aligned}
\eta_{j} & =P_{j} x e^{\lambda t}+\left(\sqrt{4 \kappa-P_{j}^{2}}-2 \xi_{0}\right) P_{j} \int_{0}^{t} e^{2 \lambda \tau} d \tau+\zeta_{1, j}, \\
Z_{j}= & \frac{\sqrt{4 \kappa-P_{j}^{2}}+i P_{j}}{\sqrt{4 \kappa-P_{j}^{2}}-i P_{j}} \\
A_{12}= & \frac{4 \kappa-P_{1} P_{2}-\sqrt{4 \kappa-P_{1}^{2}} \sqrt{4 \kappa-P_{2}^{2}}}{4 \kappa+P_{1} P_{2}-\sqrt{4 \kappa-P_{1}^{2}} \sqrt{4 \kappa-P_{2}^{2}}}
\end{aligned}
$$

here $j=1,2$. From the solution in Eq. (20), we can see its asymptotic behavior

$$
\begin{aligned}
& q_{2}(x, t) \rightarrow Z_{1} Z_{2}, \text { as } x \rightarrow+\infty \\
& q_{2}(x, t) \rightarrow 1, \text { as } x \rightarrow-\infty
\end{aligned}
$$

which shows only a phase shift $\delta_{d, 1}+\delta_{d, 2}$, here $\delta_{d, j}=$ $\arctan \left[2 P_{j} \sqrt{4 \kappa-P_{j}^{2}} /\left(4 \kappa-2 P_{j}^{2}\right)\right], j=1,2$, as from $x \rightarrow$ $+\infty$ to $x \rightarrow-\infty$. The solution in Eq. (17) with Eq. (20) describes a general scattering process of two dark solitary waves of BECs on the nonzero background, characterized by the different center velocity $V_{1}$ and $V_{2}$, respectively. From Eq. (21) we get the velocity of each soliton as $V_{j}=\left(2 \xi_{0}-\sqrt{4 \kappa-P_{j}^{2}}\right)\left(e^{\lambda t}-\lambda e^{-\lambda t} \int_{0}^{t} e^{2(\lambda \tau)} d \tau\right), j=$ 1,2 . In order to understand the nature of two solitons interaction, we analyze the asymptotic behavior of the solution in Eq. (17) with Eq. (20). Asymptotically, the two-soliton waves in Eq. (20) can be written as a combination of two one-soliton waves in Eq. (18). The asymptotic form of two-soliton solution in limits $t \rightarrow-\infty$ and $t \rightarrow \infty$ is similar to that of one-soliton in Eq. (17) with Eq. (18).

(i) Before collision (limit $t \rightarrow-\infty$ )

(a) Soliton $1\left(\eta_{1} \approx 0, \eta_{2} \rightarrow-\infty\right)$

$$
\psi \rightarrow \frac{\sqrt{\kappa} e^{i \varphi_{d}+\lambda t / 2}}{2 \mu}\left[1+Z_{1}-\left(1-Z_{1}\right) \tanh \frac{\eta_{1}}{2}\right],
$$

(b) Soliton $2\left(\eta_{2} \approx 0, \eta_{1} \rightarrow \infty\right)$

$$
\psi \rightarrow \frac{\sqrt{\kappa} Z_{1} e^{i \varphi_{d}+\lambda t / 2}}{2 \mu}\left[1+Z_{2}-\left(1-Z_{2}\right) \tanh \frac{\eta_{2}+\delta_{0}}{2}\right] .
$$

(ii) After collision (limit $t \rightarrow \infty$ )

(a) Soliton $1\left(\eta_{1} \approx 0, \eta_{2} \rightarrow \infty\right)$

$$
\psi \rightarrow \frac{\sqrt{\kappa} Z_{2} e^{i \varphi_{d}+\lambda t / 2}}{2 \mu}\left[1+Z_{1}-\left(1-Z_{1}\right) \tanh \frac{\eta_{1}+\delta_{0}}{2}\right]
$$

(b) Soliton $2\left(\eta_{2} \approx 0, \eta_{1} \rightarrow-\infty\right)$

$$
\psi \rightarrow \frac{\sqrt{\kappa} e^{i \varphi_{d}+\lambda t / 2}}{2 \mu}\left[1+Z_{2}-\left(1-Z_{2}\right) \tanh \frac{\eta_{1}}{2}\right],
$$

where the center shift of dark soliton is given by $\delta_{0}=$ $\ln A_{12}$. By analyzing the asymptotic behavior of twosoliton solution in detail, we know that there is no change of the amplitude for each soliton during collision, while one should notice that the factor $\left|Z_{j}\right|=1, j=1,2$, again. However, from Eq. (22) to Eq. (25) we find a phase exchange $\delta_{0}$ for soliton 1 and soliton 2 during collision. These results show that the collision of two dark solitons is elastic.

\section{CONCLUSIONS}

In summary, we present a family of nonautonomous soliton solutions of BECs with the time-dependent interatomic interaction in an expulsive parabolic potential. Our results show that the amplitude, width, and velocity of nonautonomous soliton can be affected by the timedependent atomic scattering length, which can be tuned by the external filed from the so-called Feshbach resonance technique. These results also provide an experimental tool for investigating the range of validity of the one-dimensional Gross-Pitaevskii equation with an abnormal expulsive parabolic potential. For the cases of both attractive and repulsive interaction, the total particles number is a conservation quantity, but the peak (dip) density of soliton can be controlled by the Feshbach resonance parameter. The soliton solutions reported here may be more realistic and leave scope for more physical explanation and application in the future. 


\section{ACKNOWLEDGMENTS}

This work is supported by Hundred Innovation Talents Supporting Project of Hebei Province of China, the NSF of China under Grant No. 10874038, the Province Natural Science Foundation of Shanxi under Grant No. 2007011007, and the Natural Science Foundation of Hebei Province under Grant No. A2008000006.
[1] N. J. Zabusky, and M. D. Kruskal, Phys. Rev. Lett. 15 (1965) 240.

[2] A. Hasegawa, Y. Kodama, Solitons in Optical Communications (Oxford University Press, Oxford, 1995).

[3] M. J. Ablowitz, P. A. Clarkson, Soliton, Nonlinear Evolution Equations and Inverse Scattering (Cambridge University Press, Cambridge, 1991).

[4] F. Dalfovo, S. Giorgini, Lev P. Pitaevskii, and S. Stringari, Rev. Mod. Phys. 71 (1999) 463.

[5] Kevin E. Strecker, Guthrie B. Partridge, Andrew G. Truscott \& Randall G. Hulet, Nature 417 (2002) 150;

L. Khaykovich, F. Schreck, G. Ferrari, T. Bourdel, J. Cubizolles, L. D. Carr, Y. Castin, C. Salomon, Science 296 (2002) 1290.

[6] L. D. Carr and Y. Castin, Phys. Rev. A66 (2002) 063602.

[7] L. Salasnich, A. Parola, and L. Reatto, Phys. Rev. Lett. 91 (2003) 080405;

G. Fibich et al., Phys. Lett. A239 (1998) 167.

[8] U. Al Khawaja, H. T. C. Stoof, R. G. Hulet, K. E. Strecker, and G. B. Partridge, Phys. Rev. Lett. 89 (2002) 200404.

[9] P. G. Kevrekidis, G. Theocharis, D. J. Frantzeskakis, and Boris A. Malomed, Phys. Rev. Lett. 90 (2003) 230401.

[10] Lu Li, Zaidong Li, Boris A. Malomed, Dumitru Mihalache, and W. M. Liu, Phys. Rev. A 72 (2005) 033611; $\mathrm{Lu} \mathrm{Li}$, Boris A. Malomed, Dumitru Mihalache, and W. M. Liu, Phys. Rev. E 73 (2006) 066610.

[11] L. Wu, J.-F. Zhang, and L. Li, New J. Phys. 9 (2007) 69.

[12] S. L. Cornish, S. T. Thompson, and C. E. Wieman, Phys. Rev. Lett. 96 (2006) 170401.

[13] R. Dum, J. I. Cirac, M. Lewenstein, and P. Zoller, Phys. Rev. Lett. 80 (1998) 2972.

[14] S. Burger, K. Bongs, S. Dettmer, W. Ertmer, K. Sengstock, A. Sanpera, G. V. Shlyapnikov, and M. Lewenstein, Phys. Rev. Lett. 83 (1999) 5198;

J. Denschlag et al., Science 287 (2000) 97.

[15] A. D. Jackson, G. M. Kavoulakis, and C. J. Pethick, Phys. Rev. A 58 (1998) 2417.

[16] Th. Busch and J. R. Anglin. Phys. Rev. Lett. 84 (2000) 2298 ;

C. K. Law, C. M. Chan, P. T. Leung, and M.-C. Chu, Phys. Rev. Lett. 85 (2000) 1598;

Biao Wu, Jie Liu, and Qian Niu, Phys. Rev. Lett. 88 (2002) 034101.

[17] J. C. Bronski, L. D. Carr, B. Deconinck, and J. N. Kutz, Phys. Rev. Lett. 86 (2001) 1402;

D. L. Feder, M. S. Pindzola, L. A. Collins, B. I. Schneider, and C. W. Clark, Phys. Rev. A 62 (2000) 053606.

[18] V. N. Serkin, A. Hasegawa, and T. L. Belyaeva, Phys. Rev. Lett. 98 (2007) 074102.

[19] H. H. Chen and C. S. Liu, Phys. Rev. Lett. 37 (1976)
693; Phys. Fluids 21 (1978) 377

[20] V. V. Konotop, Phys. Rev. E 47 (1993) 1423;

V. V. Konotop, O. A. Chubykalo, and L. Vázquez, Phys. Rev. E 48 (1993) 563.

V. V. Konotop, Theoretical and Mathematical Physics, 99 (1994) 687.

[21] Qiu-Yan Li, Zai-Dong Li, Shu-Xin Wang, Wei-Wei Song, Guangsheng Fu, Opt. Commun. 282, 1676 (2009).

[22] V. N. Serkin and A. Hasegawa, Phys. Rev. Lett. 85 (2000) 4502.

[23] V. N. Serkin, et al., JETP Lett. 74 (2000) 573;

V. N. Serkin, JETP Lett. 72 (2000) 89;

V. N. Serkin, et al., IEEE J. Quantum Electron. 8 (2002) 418.

[24] Lei Wu, Jie-Fang Zhang, Lu Li, C. Finot, and K. Porsezian, Phys. Rev. A 78 (2008) 053807

[25] J. L. Roberts, N. R. Claussen, James P. Burke, Jr., Chris H. Greene, E. A. Cornell, and C. E. Wieman, Phys. Rev. Lett. 81 (1998) 5109;

J. Stenger, S. Inouye, M. R. Andrews, H.-J. Miesner, D. M. Stamper-Kurn, and W. Ketterle, Phys. Rev. Lett. 82 (1999) 2422.

[26] K. Staliunas, S. Longhi, and G. J. de Valcárcel, Phys. Rev. Lett. 89 (2002) 210406.

[27] P. Engels, C. Atherton, and M. A. Hoefer, Phys. Rev. Lett. 98 (2007) 095301.

[28] F. Kh. Abdullaev, A.M. Kamchatnov, V.V. Konotop, and V. A. Brazhnyi, Phys. Rev. Lett. 90 (2003) 230402.

[29] D. E. Pelinovsky, P. G. Kevrekidis, and D. J. Frantzeskakis, Phys. Rev. Lett. 91 (2003) 240201.

[30] P. G. Kevrekidis and D. J. Frantzeskakis, Mod. Rhys. Lett. B 18 (2004) 173.

[31] V. A. Brazhnyi and V. V. Konotop, Mod. Rhys. Lett. B 18 (2004) 627.

[32] Z. X. Liang, Z. D. Zhang, and W. M. Liu, Phys. Rev. Lett. 94 (2005) 050402.

[33] V. M. Pérez-García, V. V. Konotop, and V. A. Brazhnyi, Phys. Rev. Lett. 92 (2004) 220403.

[34] L. Li, Z. H. Li, S. Q. Li, and G. S. Zhou, Opt. Comm. 234 (2004) 169.

[35] S. Q. Li, L. Li. Z. H. Li, and G. S. Zhou, J. OPt. Soc. Am. B 21 (2004) 2089.

[36] Z. Y. Xu, L. Li, Z. H. Li, and G. S. Zhou, Phys. Rev. E 67 (2003) 026603.

[37] R. Hirota, J. Phys. Soc. Jpn. 51 (1982) 323;

Zai-Dong Li, Qiu-Yan Li, Xing-Hua Hu, Zhong-Xi Zheng, Yubao Sun, Ann. Phys. (N.Y.) 322 (2007) 2545.

[38] L. Li, Z. H. Li, Z. Y. Xu, G. S. Zhou, and K. H. Spatschek, Phys. Rev. E66, 046616 (2002). 